\title{
Immediate Essential Care for New-borns in the Six Hospitals of Conakry: Knowledge and Practices of Providers
}

\author{
Soumah Aboubacar Fode Momo ${ }^{1, ~}{ }^{*}$, Keita Massa ${ }^{1}$, Conte Ibrahima ${ }^{1}$, Camara Soriba Naby ${ }^{2}$, \\ Diallo Abdourahamane ${ }^{1}$, Leino Watanasse ${ }^{3}$, Balde Ibrahima Sory ${ }^{1}$, Sy Telly ${ }^{1}$ \\ ${ }^{1}$ Department of Gynaecology and Obstetrics, Ignace Deen National Hospital, Faculty of Sciences and Health Technic, Gamal Abdel Nasser \\ University of Conakry, Conakry, Guinea \\ ${ }^{2}$ Department of Visceral Surgery, Friendship Hospital Sino Guinean, Faculty of Sciences and Health Technic, Gamal Abdel Nasser University \\ of Conakry, Conakry, Guinea \\ ${ }^{3}$ Department of Gynecology and Obstetrics, Donka National Hospital, Faculty of Sciences and Health Technic, Gamal Abdel Nasser \\ University of Conakry, Conakry, Guinea
}

Email address:

aboubacarfodemomo@gmail.com (S. A. F. Momo)

${ }^{*}$ Corresponding author

\section{To cite this article:}

Soumah Aboubacar Fode Momo, Keita Massa, Conte Ibrahima, Camara Soriba Naby, Diallo Abdourahamane, Leino Watanasse, Balde Ibrahima Sory, Sy Telly. Immediate Essential Care for New-borns in the Six Hospitals of Conakry: Knowledge and Practices of Providers. Journal of Gynecology and Obstetrics. Vol. 9, No. 5, 2021, pp. 172-177. doi: 10.11648/j.jgo.20210905.17

Received: September 4, 2021; Accepted: September 29, 2021; Published: October 15, 2021

\begin{abstract}
The aim of this work was to analyze the organization and execution of immediate essential care for newborns in referral hospitals in Conakry. Immediate essential newborn care is a key component for a maternity hospital. lower risk, but often overlooked by health providers. Methodology: This was a descriptive and analytical cross-sectional study carried out through an observation of the practices of immediate care for newborns and the individual interview of providers in six referral hospitals in urban areas of Conakry, from April 1 to June 30,2020. We have carried out an exhaustive recruitment of all providers working in the delivery room and meeting the inclusion criteria. We used the checklist, the written questionnaire for individual interviews and the provider observation grid. Provider knowledge and practice was observed during immediate neonatal care. The independent variables were socio-demographic factors and personal and institutional factors. Statistical comparisons were made using the chi-square test. the differences were considered significant for $p<0.05$. Results: In this study 69 providers agreed to participate in the study, with a response rate of $100 \%$, the largest proportion $61 / 69(88.4 \%)$ were midwives, In terms of knowledge: $56,5 \%$ of providers cited all the equipment and consumables to be prepared at the time of childbirth, $94.2 \%$ on thermal protection, $82.6 \%$ on the components of immediate newborn care, In terms of skills: $84.1 \%$ of our providers recognized newborns needing resuscitation and $15.9 \%$ were competent on this procedure, skin-to-skin contact was achieved in $68.1 \%$. On the other hand, thermal protection is statistically linked to knowledge of the normal temperature of the newborn $\mathrm{p}=0.015$. For the prevention of infection, there is a significant relationship with the training of providers $\mathrm{p}=0.001$. Conclusion: Globally, newborn care providers have gaps in the knowledge and practice of the immediate care they offer newborns, this situation can only be improved by close monitoring of providers.
\end{abstract}

Keywords: Knowledge, Practices, New-borns, Immediate Care

\section{Introduction}

Neonatal mortality remains a public health problem although the trend is decreasing [1]. The countries of subSaharan Africa have the highest rates. Indeed the number of neonatal deaths was 3 million in 2011 and the neonatal mortality rate equal to 22 per 1000 live births in the world [2]. In this part of the African continent there are 1.16 million deaths of newborns during the first month of life and one million cases of stillbirths. Up to half a million African babies die the same day they are born [3]. 
In Guinea, the neonatal mortality rate was estimated at 35 per 1000 live births in 2010 [4]. The determinants of causes of unacceptable neonatal death are multifactorial:

There are the direct causes, which are medical pathologies, the main ones of which are: Severe infections which represent $38 \%$, in particular pneumonia / septicemia $28 \%$, tetanus $6 \%$ and diarrhea $4 \%$; Prematurity 25\%; 24\% asphyxiation; Congenital malformations $6 \%$ [3];

At the center of all these pathologies is low birth weight or low birth weight, which is the third leading cause of neonatal death [5]. But one should not lose sight of the very quality of newborn care; quality care requires the provider to have a good level of theoretical knowledge and to perform procedures according to standards.

The third possible determinant of neonatal deaths concerns the inadequacies that may exist in the care environment: this concerns the availability of equipment, consumables and an effective organization of care in the birthing room.

Finally, there is the level of compliance with infection prevention rules in the room and during the care provided to these fragile beings.

In Guinea, as everywhere in Sub-Saharan Africa, many actions are carried out every day to improve the survival of newborns.

Our country is one of the six (06) low-income countries that have succeeded in significantly reducing the rate of neonatal deaths; But, according to a WHO report, subSaharan Africa is still the most dangerous region in the world for an unborn child [6]. There are no reliable data on the practice and organization of essential newborn care in maternity hospitals in Conakry, such data can allow the implementation of simple, safe and effective corrective measures, and that can be undertaken by health providers to prevent or treat most newborn problems [7]. These actions consist of ensuring immediate and complete drying, skin-to-skin contact of the newborn with the mother, clamping and then sectioning the cord after the first few minutes after childbirth, early latching and latching. 'exclusive breastfeeding, eye care, administration of vitamin $\mathrm{K}$, and education of the mother on danger signs for the newborn [8]. Care in the delivery room is the key to the survival of these newborns. They are defined as "the set of gestures and acts to be performed at the time of birth and up to six (6) weeks of life, to ensure the survival of the newborn" [9].

This study will therefore allow us to know the environment, the equipment and the practices and knowledge of the immediate essential care administered to newborns in the five (05) hospitals of Conakry in order to take corrective measures.

The objectives of this study were: to describe the sociodemographic and professional characteristics of providers who provide essential newborn care, to identify the availability of equipment and consumables, to assess the knowledge and practices of providers in immediate newborn care.

\section{Methodology}

\subsection{Study Framework}

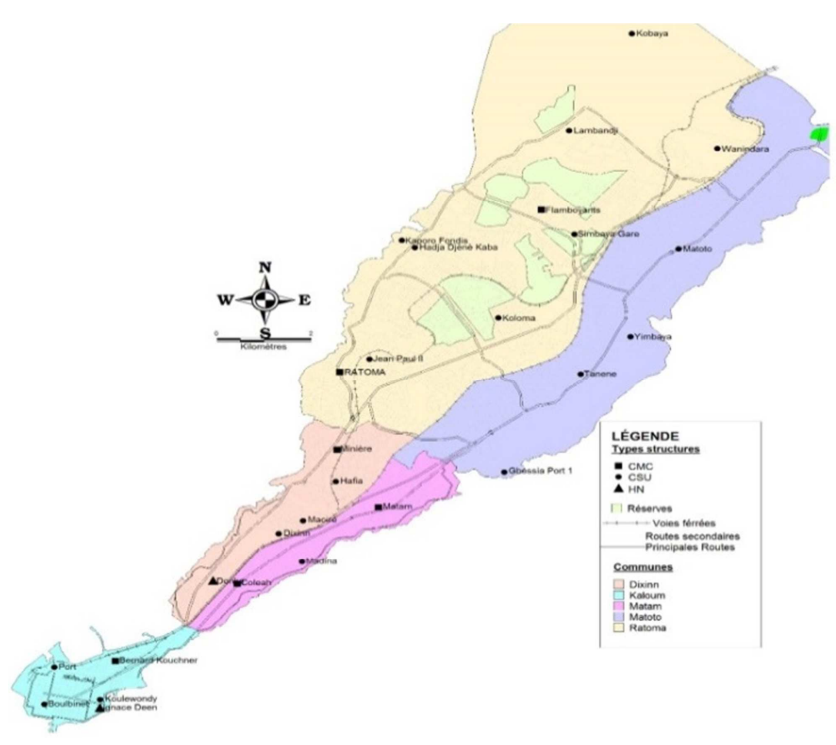

Figure 1. Mapping of the city of Conakry.

The study took place in the city of Conakry, capital of Guinea, a coastal country with an area of $245,857 \mathrm{~km}^{2}$. According to the data [4], the Guinean population was estimated at 12,730,977 inhabitants in 2012. Public healthcare structures are organized into three (03) levels according to data from the 2012 DHS:

1. The first level corresponds to the health center

2. The second level is represented by the improved health center with a surgical branch, the communal medical centers, the Prefectural and Regional Hospital Center. They serve as a reference and recourse to the health posts, health center;

3. The third level is made up of the University Hospital Center (CHU). It is the highest reference level for specialist care.

The study sites were: The Ignace Deen University Hospital Center, third level of reference in the health pyramid; four Communal Medical Centers thus constituting the second level in the Guinean health pyramid. Including two with surgical antenna (CMC of Matam, Ratoma, Bernard couchner), Coléah without surgical antenna

\subsection{Type and Period of Study}

This was a descriptive and analytical cross-sectional study carried out through an observation of the practices of immediate newborn care in referral hospitals in urban areas of Conakry from April 1 to June 30, 2020.

\subsection{Inclusion and Non-inclusion Criteria}

\subsubsection{Inclusion Criteria}

The following were included in this study:

1. Providers working in the delivery rooms of the maternity hospitals selected for the study.

2. Any maternity care provider who has already provided 
essential newborn care

Verbal consent to participate in the study will be obtained from any included provider.

\subsubsection{Non-inclusion Criteria}

Trainees and any other health worker passing through the departments concerned at the time of the study will not be surveyed.

\subsection{Sampling and Calculation of the Sample Size}

We systematically included all delivery room providers who met the inclusion criteria, allowing us to have 69 providers.

Data collection tools and procedures:

All providers were surveyed by observation and direct individual interviews with the same questionnaire.

In addition to individual interviews, those who will still be stationed in the delivery room will be the subject of direct observation during care, two observations were made for each provider:

1. The first observation: if the service provider performs all the actions according to the standard, he is said to be competent.

2. The second observation: if the provider does not carry out the actions in an orderly fashion with some verification criteria not met, he is said to be noncompetent, he is given the Fed back before moving on to the second patient.

We verified the existence of care and infection prevention equipment in each department by direct observation using a standard list of equipment.

The instrument for collecting the data will consist of three main parts:

a. The first part which will be a checklist of essential care equipment, infection prevention and service organization.

b. The second part will be a written questionnaire for individual interviews with providers on their knowledge of the equipment and practice of essential newborn care

c. The last part will be an observation grid for providers while they will care for newborns

\subsection{Study Variables}

The dependent variable was the knowledge and practice of health care providers in immediate neonatal care. The independent variables were socio-demographic factors and personal and institutional factors.

\subsection{Operational Definitions}

Good knowledge:

If the service provider cites all the materials and where some of the knowledge

Poor knowledge

If the provider does not cite any knowledge material

Good practices or competence

When the health care provider achieves $85 \%$ performance in performing procedures according to standards

Bad practice or no competence

When the health care provider does not achieve an $85 \%$ performance

\subsection{Analysis Plan}

Descriptive analyzes were carried out on the sociodemographic and professional characteristics of the service providers; The availability of the heat source; The questions relating to knowledge about infection prevention and the organization of the service were calculated and noted yes and no, general knowledge was calculated by the sum of the yeses of each variable over all the observed variables multiplied. by 100; knowledge of essential newborn care equipment and practice; the observation of The practice of essential newborn care was noted by assigning $(\mathrm{C})$ : competent if the procedure was correctly performed, (NC): not competent if it did not, NA not applicable if the procedure is not feasible.

An analytical study of our variables was carried out, on the state of the training received, thermal protection, breastfeeding and prevention of infection, knowledge of the normal temperature of the newborn and thermal protection, The data were analyzed using Epinfo 1.1.2 software, which allowed us to calculate the frequency. Statistical comparisons were made using the Chi2 test. The differences were considered significant for $\mathrm{p}<0.05$

\section{Results}

For this study, we selected 69 providers from the four (04) municipal medical centers in the city of Conakry: Bernard Couchner 10 providers (14.49\%), Ratoma15 (21.74\%), Matam $19(27.54 \%))$, Coleah $8(11.59 \%)$ and the maternity hospital Ignace Deen 17 (24.64\%).

Table 1. Sociodemographic and professional characteristics of providers.

\begin{tabular}{lll}
\hline Qualification of providers & Number & Percentage \\
\hline Midwife & 61 & 88,4 \\
Health technician acting as a midewife & 4 & 5,8 \\
Recycled acting as nurse & 3 & 4,3 \\
Nurse acting as a midwife & 1 & 1,4 \\
Total & 69 & 100 \\
Experience in the work & & \\
One year & 13 & 18,8 \\
2-5 years & 33 & 47,8 \\
6-11 years & 12 & 17,4 \\
Plus, de 12 years & 11 & 15,9 \\
Total & 69 & 100 \\
Immediate Essential Care Training Newborns & & \\
between 2018-2019 & & \\
Soins obstétricaux et néonatals d'urgences de & 11 & 15,9 \\
base & 25 & 36,2 \\
Soins essentiels aux nouveau-nés & 33 & 47,8 \\
Aucune formation & 69 & 100 \\
Total & & \\
Practice of post-training care & 43 & 62,3 \\
basic emergency obstetric and neonatal care & 26 & 37,7 \\
Newborn care not provided & 69 & 100 \\
Total & & \\
\hline
\end{tabular}


All of our study sites either $100 \%$ owned the heat source or from city power, generator set or solar panel to operate the heat lamp.

The prevention of infection which consists in washing the hands either with soap and water or the hydro-alcoholic solution before and after the procedure, in our series 50 of our providers $(72.5 \%)$ washed their hands. hands with soap and water, $46(66.7 \%)$ used the three bins for sorting waste, $68(98.6 \%)$ wore the clean and or sterile glove, $59(85.5 \%)$ the sleeved glove during childbirth, the bonnet was used by $62(89.9 \%)$, face mask or bib was under used during the procedure $25(36.2 \%)$, the apron in $41(59.4 \%))$, waterproof footwear in $51(73.9 \%)$. All providers were $100 \%$ competent in preparing the decontamination solution

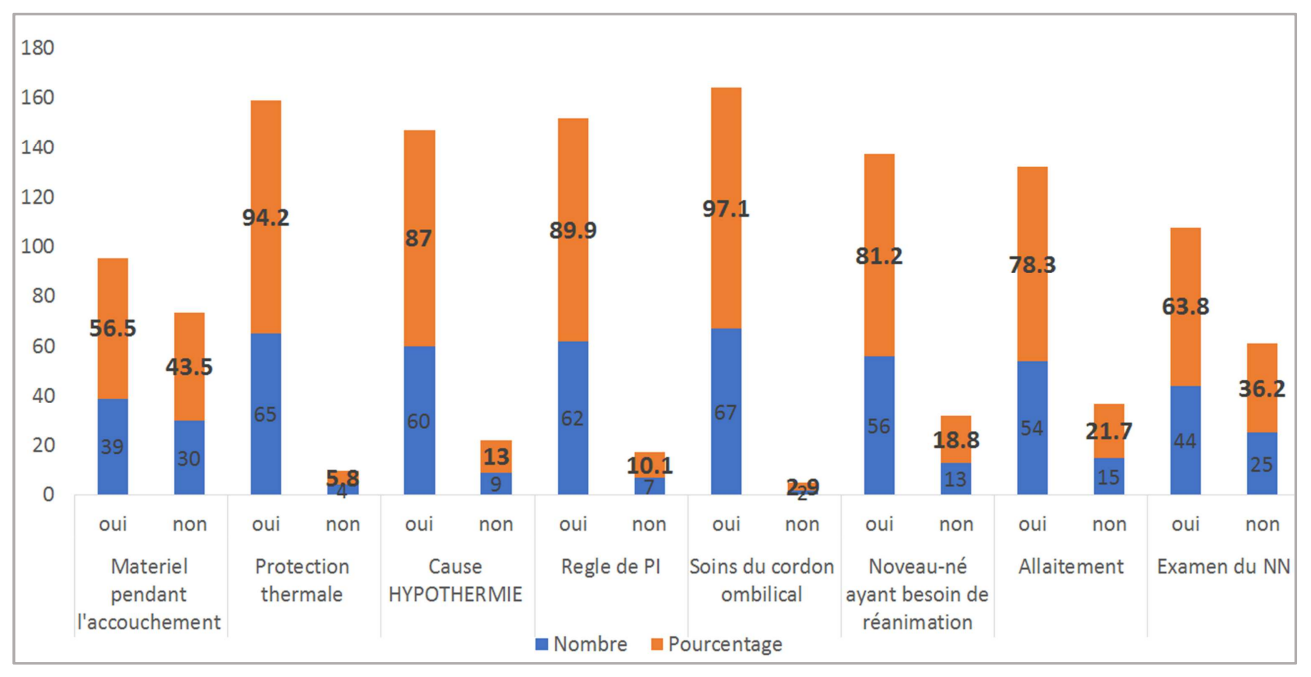

Figure 2. Knowledge about immediate care for newborns.

\section{Skills}

We raised provider skills during the administration of immediate newborn care: 58 providers (84.1\%) were proficient in identifying newborns needing resuscitation, only $11(15.9 \%)$ were competent for the procedure, the majority were uncompetent $58(84.1 \%)$, for hypothermia almost all providers $67(97.1 \%)$ were competent for the prevention of hypothermia versus $2(2.9 \%)$. Skin-to-skin contact was competently performed correctly by our providers 47 (68.1\%) against $22(31.9 \%)$. More than half of our providers were skilled in performing delayed cord clamping.

The practice of caring for newcomers, in our study $71 \%$ of our providers were competent in building confidence in the parturient through greeting and presentation; 92.8\% consulted the antenatal consultation booklet, $47.8 \%$ asked for the progress of the pregnancy, $89.9 \%$ were competent in the preparation of the delivery table and equipment to bring according to their use, 69, 6\% wore personal protective equipment; $53.6 \%$ were skilled in episiotomy repair and $91.3 \%$ were skilled in postpartum care.

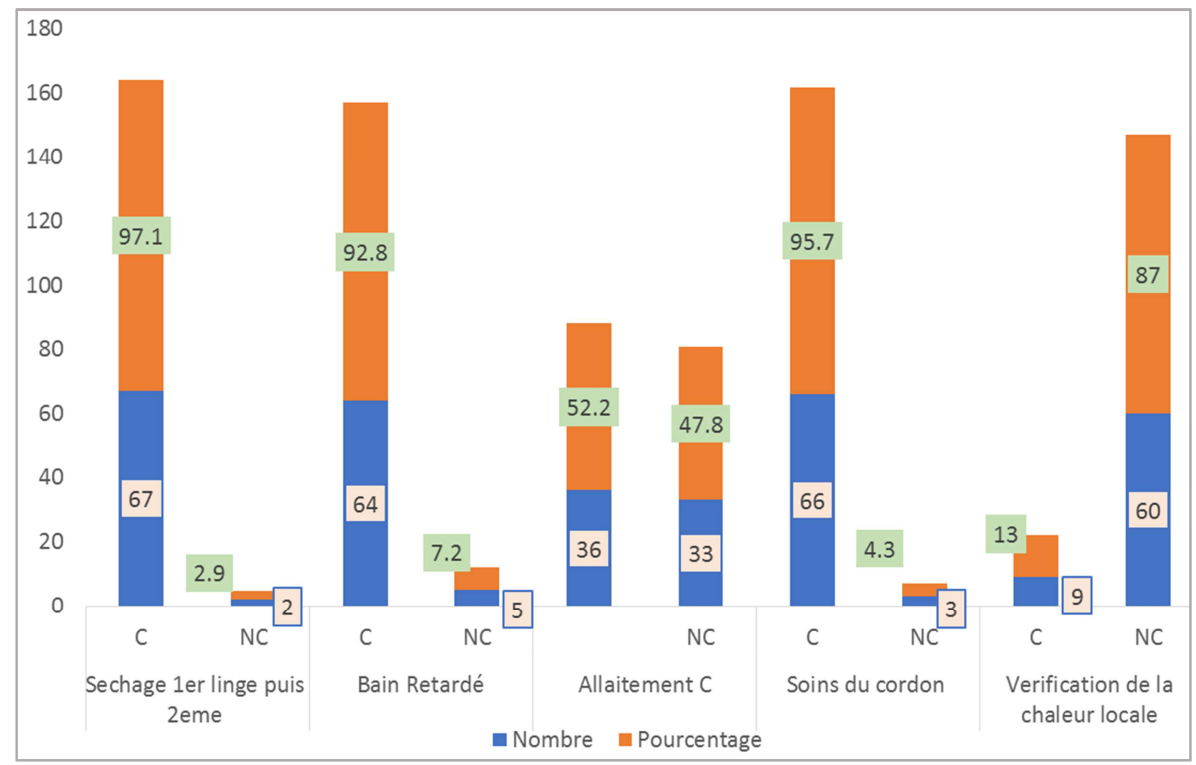

Figure 3. Skills during cares. 
Newborn examination, in our study our providers were not proficient in comprehensive newborn examination $64(92.8 \%)$ vs. $5(7.2 \%)$ competent providers, this was the challenge major for this study, on the other hand $67(97.1 \%)$ were competent in taking parameters of the newborn 67 (97.1\%), eye care in $68(98.6 \%)$, bathing delayed for 24 Hours to avoid hypothermia $64(92.8 \%)$, clothed the newborn had become the reflex in $64(92.8 \%)$, the infection prevention procedure was observed after the procedure $65(94,2 \%)$.

Statistical tests

All the trained providers applied thermal protection of the newborn, on the other hand $12.13 \%$ of the untrained providers did not do so exposing these newborns to the risk of neonatal hypothermia, we had not put a significant relationship between training and thermal protection $\mathrm{P}=0.47$, for $\operatorname{chi} 2=4.63$.

It was found that $75 \%$ of providers who did not perform thermal protection did not know the normal temperature of the newborn. This ignorance exposes them to a risk of hypothermia $\mathrm{P}=0.015$, for $\mathrm{chi} 2=9.8$.

It is found that, $47.82 \%$ of providers were not trained in infection prevention and $90.9 \%$ of these untrained providers had not implemented prevention of cord infections in newborns. This practice exposes newborns to a major risk of infection to the umbilical cord. We found a statistically significant link between training and prevention practice. $\mathrm{P}=0.001 \mathrm{P}=0.001$, for $\operatorname{chi} 2=5.88$.

It can be seen that $52.17 \%$ of providers started breastfeeding within one minute of giving birth. This proportion drops to $33.33 \%$ for untrained people and rises to $69.44 \%$ when the provider is trained. We found a statistically significant relationship $\mathrm{P}=0.003$ for chi $2=8.49$.

The training had an impact on the practice of infection prevention, $72.5 \%$ of our providers washed their hands, which is the first barrier to infection control before and after the procedure, we noted a very significant relationship between formation and prevention of infection $\mathrm{P}=0.001$ for chi $2=12.16$.

\section{Discussion}

Our cross-sectional study carried out in four maternity wards of communal medical centers and one maternity unit at the National Ignace Deen Hospital in Conakry.

Overall, sixty-nine providers agreed to participate in the study, with a response rate of $100 \%$; the largest proportion came from the Matam CMC 27.54\%.

Sociodemographic and professional characteristics of service providers (Table 1). The largest proportion of our sample 61/60 (88.4\%) were midwives, who have work experience between 25 years were $47.8 \%$ the result in our study was higher than that of Hagos Tasew et al. In Ethiopia was 25.7\% [10], more than half of our providers received specific training on essential newborn care $52.12 \%, 15.9 \%$ received training on immediate infant care. newborns during SONUB training; The result of our study was lower than that reported by Faridullah Atiqzai in Afghanistan 20.7\% [11]; this difference could be explained in part by the frequent movement of providers, but also the retirement and $62.3 \%$ provided essential care for newborns against $37.7 \% .100 \%$ in our study sites either through the city current, the generator, or the solar panel to this is added the functional heating lamp, unlike the study [10] respectively $67.6 \%, 75 \%$ lacked the heat source.

Infection prevention and organization (Text 3 ), all the sites of our study had in the procedure rooms at the time of the investigation a source of water, soap, decontamination solution.

All providers knew how to make the $100 \%$ decontamination solution, the majority 50/69 (72.5\%) washed their hands with soap and water before the procedure, $98.6 \%$ wore gloves, $89,9 \%$ the cap $36.2 \%$ the bib or face mask, $59.4 \%$ the apron, $73.9 \%$ the waterproof boot, the results in our study were superior to those of Hagos et al. [10], in Ethiopia who reported $65.9 \%$ hand washing, $74 \%$ wearing gloves, wearing an apron $73.7 \%$, bibs $60.3 \%$, this difference could be explained in part by the training and the year of the studies, the practice of infection prevention according to standards, during childbirth and immediate newborn care are essential to reduce infections associated with newborn care, we have put a very significant relationship between the training of newborns. providers and the practice of infection for $\mathrm{p}<0.001$.

Carrying out immediate care for newborns requires knowledge and practice of the equipment involved in the procedure (figure 1). In our study, more than half of our providers $56.5 \%$ knew the equipment to prepare during childbirth, this observation could be explained by the qualification of our providers but also by the number of years of experience, 94, 2\% knew the importance of thermal protection, the result of our study was superior to that of Faridullatt Atiqz et al. in Afghanistan, which reported 57.4\% [11], this difference could be explained by the study population, the length of time in the job, but also by training. Based on this observation, we have demonstrated a significant relationship between knowledge of the normal temperature of the newborn and thermal protection for $\mathrm{p}<0.015$, on the other hand there is no significant relationship between training and thermal protection. $p<0.47$.

Overall, training had a positive influence in our study on the knowledge of newborns needing resuscitation $81.2 \%$, breastfeeding $78.3 \%$, examination of the newborn $63.8 \%$. (Figure 1)

The childbirth would require preparation of providers on all levels, building confidence through the greeting was ensured in $71 \%$ in our series, $92.8 \%$ consulted the diary of the parturient, the challenges remain to be overcome in relation to questioning about the course of the pregnancy only less than half of our providers $47.8 \%$ were competent; $89.9 \%$ of our providers, prepared the delivery table and the material according to their use during the delivery procedure, $69.6 \%$ wore the personal protective equipment before the procedure, $53.6 \%$ were competent on episiotomy repair and $91.3 \%$ were competent in postpartum care.

Newborn care requires enough skills, which is why in our 
series, $84.1 \%$ of our observed providers were competent in identifying newborns in need of resuscitation, this indicator represents a challenge for this study because only $15.9 \%$ were competent to perform this procedure, Joseph de GraftJohnson et al in a study in the six countries of Sub-Saharan Africa, noted the similar finding [12] by for the prevention of hypothermia only 9 providers $(13 \%)$ were competent at finding local heat by palpating the heel of the newborn versus $60(87 \%)$ were not competent; and on immediate drying in a first dry cloth then the second (figure 2), 97.1\% were competent; skin-to-skin contact was carried out in $68.1 \%$ of cases, in our study our providers were competent in $92.8 \%$ on the merits of delaying the first bath in the first 24 hours, on the accompaniment of childbirth at the start of breastfeeding in the minutes following birth more than half of our providers were competent $52.2 \%$, the similar observation was observed by Ribka Amsalu et al [13] in Somalia with the following proportions immediate drying $98 \%$, delayed bathing $99.2 \%$, skin-to-skin contact $8.6 \%$, we demonstrated a significant difference between the training received and the start of breastfeeding for $\mathrm{p}=0.003$., $95.7 \%$ were competent in the management of umbilical cord care,: It is noted that, $47.82 \%$ of providers have not been trained in Infection prevention and $90.9 \%$ of these providers are not trained. had not applied prevention of cord infections in newborns.

This practice exposes newborns to a major risk of infection through the umbilical cord. We found a statistically significant link between training and the practice of prevention, a single provider was competent, i.e. $1.4 \%$, on testing for anal imperforation, almost all of our providers, i.e. $97.1 \%$, took the newborn parameters (Height, Weight, Head Perimeter), they were competent on eye care by applying tetracycline ointment $98.6 \%$, hand washing after the procedure $94.2 \%$, our providers were not competent in the comprehensive examination of the newborn $64(92.8 \%)$ vs. 5 (7.2\%) competent providers, this was the major challenge for this study, vs. 67 (97.1\%) were competent in taking newborn parameters $67(97.1 \%)$, eye care in $68(98.6 \%)$, bathing delayed for 24 hours to avoid hypothermia 64 (92.8\%), dressed the new -born had become the reflex in 64 (92.8\%), the procedure for prevention of infection was observed after the procedure $65(94.2 \%)$.

\section{Conclusion}

The immediate care of newborns is an important stage, it necessarily involves knowledge of the appropriate procedures and the competence of health providers.

\section{References}

[1] OMS, Save The Children. La mortalité néonatale baisse trop lentement surtout en Afrique. PLoS Medicine. 2011. http://www.who.int/mediacentre/news/releases/2011/newborn deaths_20110830/fr/ 30 juillet 2014.

[2] OMS. Statistiques sanitaires mondiales 2013. http://apps.who.int/iris/bitstream/10665/82056/1/9789242564 587 fre.pdf, consulté le 30 juillet 2014.

[3] Lawn, J., and K. Kerber. "Donnons sa chance à chaque nouveau-né de l'Afrique: données pratiques, soutien programmatique et de politiques pour les soins du nouveau-né en Afrique, éds." Partenariat pour la santé maternelle, néonatale et infantile, Cape Town (2006). P: 5, 16.

\section{[4] EDS-MICS GUINEE 2012.}

[5] USAID, BASICS, MSP, DRS. Guide du formateur des prestataires. Soins essentiels aux nouveau-nés 2008 (Sénégal). http://www.basics.org/documents/Guide_du_formateur_des_p restataires_Soins_Essential_aux_Nouveaus-ne.pdf consulté le 2 août $201 \overline{4}$.

[6] OMS. Une chance pour les nouveau-nés d'Afrique. 22 Novembre 2006 http://www.who.int/mediacentre/news/releases/2006/pr70/fr/ consulté le 5 août 2014.

[7] Darmstadt, Gary L, Zulfiqar A Bhutta, Simon Cousens, Taghreed Adam, Neff Walker, Luc de Bernis, "Evidence-based, cost-effective interventions: how many newborn babies can we save? The Lancet 365. 9463 (2005): 977-988.

[8] OMS. Les soins du nouveau-né. 2014 $\mathrm{http}: / /$ www.who.int/maternal_child_adolescent/topics/newbor n/care at_birth/fr/ consulté le 2 août 2014.

[9] République du Mali, Ministère de la Santé. MANUEL DE REFERENCE Soins Essentiels du Nouveau-Né, 2009. http://www.basics.org/documents/15-Reference-Manual-forENC-at-Facility-Level.pdf consulté le 27 septembre 2014.

[10] Hagos Tasew, Tsega Teshale, Degena Bahrey, Teklewoini Mariye, Girmay Teklay, Soins immédiats du savoir, des pratiques et des facteurs associés chez le nouveau-né parmi les prestataires de soins de santé dans les établissements de santé de la zone nord-ouest Tigray, Ethiopie 2018 BMC Res Notes. 2019; 12: 427, doi: 10.1186/s13104-019-4465-z [PubMed].

[11] Faridullah Atiqzai, Partamin Manalai, Sher Shah Amin, Karen M. Edmond, Malalai Naziri, Mohammad Samim Soroush, Sharmina Sultana, Khaksar Yousufi, Thomas Van den Akker, Jelle Stekelenburg, et Hanna Tappis. Qualité des soins essentiels au nouveau-né et de la réanimation néonatale dans les établissements de santé en Afghanistan: évaluation transversale. BMJ Open 2019; 9 (8): e030496, doi: 10.1136/bmjopen-2019-030496 [PubMed].

[12] Joseph de Graft-Johson, Linda Vesel, Heather E Rosen, Barbara Rawlins, Stella Abwao, Goldy Mazia, Robert Bozsa, Winifrede Mwebesa, Neena Khadka, Rsemary Kamunya, Ashebir Getachew, Gaudiosa Tbejuka, Jean Pierre Rakotovao et Alemnesh Tekleberhan. Evaluation observationnelle transversale de la qualité des soins du nouveau-né immédiatement après la naissance dans les établissements de santé de six pays d'Afrique Subsaharienne BMJ Open 2017; 7 (3): e014680 doi: 10.1136/bmjopen-2016-014680.

[13] Ribka Amsalu, Catherine N. Morris, Kingsley Chukwumalu, Michelle Hynes, Shehryar Janjua, Alexia Couture, Aimee Summers, Amy Cannon, Erin N. Hulland et Sabine Baunach. Pratique de soins essentiels au nouveau-né dans quatre centres de soins de santé primaires dans la les zones touchées par le conflit de Bossaso, en Somalie: une Etude transversale Confl Santé 2019; 13: 27 doi 10.1186/s13031-019-0202-4 [PubMed]. 